\title{
The Research progress of Conversion of Land Use and its Effects at
}

\section{Small region extent}

\author{
Kun Dong ${ }^{1, a}$, Xue Ru Zhang ${ }^{2, b}$ \\ ${ }^{1}$ Huihua College of Hebei Normal University, Shijiazhuang, 050091,China; \\ ${ }^{2}$ Chongqing JiaoTong University, Chongqing, 400074, China \\ a1942553055@qq.com; bzhangxueru5@126.com
}

\section{Keywords: CLUE - S model LUCC Composition}

Abstract: The land use change is an important research field of landscape ecology, through the model simulation of land use change tendency is one of the main research content of this field. CLUE - S model as one of the model can well simulate the land use change of small scale, it has multiple areas at home and abroad, and is widely used in the study of land use change. This paper discusses on the domestic and foreign scholars on CLUE - S, the application of the mode. On the basis of CLUE - S model are analyzed in detail, and the development direction of the model is discussed.

\section{preface}

LUCC is the important content of the current research of global change, and the simulation and analysis of land use change become one of the core content of LUCC research. Regional land use change is an extremely complex geographic process, establishes the land use change model is the in-depth understanding of regional land use change causes, process, and predict the future development trend of important means, is a important method in LUCC and global research. In the past 20 years, different scholars on LUCC has carried out a lot of research. In recent 10 years, the LUCC space model has got rapid development. This kind of model with the aid of computer technology, the RS and GIS technology to explore on LUCC study a certain scale of dominance of spatial distribution pattern and characteristics, and analysis the natural and human driving factors on LUCC effects of spatial heterogeneity [1]. In this kind of space models, CLUE - S model is relatively mature one.

\section{CLUE - S model application progress}

CLUE - S (Conversion of Land Use and its Effects at Small region extent) model is the wageningen university in the Netherlands by a f. h. Verburg scientists such as "Land Use change and influence" team in early CLUE model on the basis of the development of. Through multiple case of empirical research, the team began in July 2002 to the world of peer recommendation CLUE - S model [2-3]. CLUE - S model since its launch, many scholars at home and abroad respectively using this model to solve the problem of land use in different areas of the world made a great deal of research work.

\section{International utilization of the model}

Koen p. Overmars [4] , in northeast of Luzon in the Philippines, KaGeYang river basin as study area, using CLUE - S model to simulate the land use pattern in the study area, and the simulation 
results are analyzed in detail.

Verburg, P.H, used the CLUE - S model to simulated the Europe over the next 30 years of land use pattern change is simulated. In this paper, the author based on the land in Europe, under the demand of the global collaboration, economic globalization and marketization of the continent, the regional integration and so on four kind of situation, simulated the situation of land use change over the next 30 years[5] .

Wytse Engelsman used CLUE - S model to simulated the land use change of the Selangor river from 1999 to 2014. This study selected the 15 driving factor, respectively in the area are the three different scenarios simulation [6].

Vincent Orekan [7] in view of the west African region, such as serious land degradation condition, the "west Africa scarce water resources effective management comprehensive evaluation" (IMPETUS) project, under the support of 900 square kilometers of benin area as the research area, selected the macro social economic factors, spatial location factor as the driving parameter, using the CLUE -s model to simulate the changes of land use in this region.

Castella, j.c. using the CLUE - S model and Agent - -based models (ABM) model, analysised and simulated the Vietnam mountain land use pattern change [8].

\section{Domestic utilization of the model}

After 2004, the domestic scholars use CLUE - S model, in view of the different parts of the land use situation in our country has carried out a lot of research work in succession.

DuanZeng qiang [9] etc.strengthen the section such as the CLUE - S model is improved, the introduction of dynamic computing neighborhood analysis factor, can be spontaneous process of land use change, the self-organizing process simulated, and the competition between the land use type can also build different simulation scheme based on the study area. CLUE - SII then apply the improved model of Beijing's haidian district from 1991 to 2001 was carried out in land use change program simulation.

ShengSheng [10] applications such as CLUE - S model, with support from the relevant data such as Landsat TM image, to the nanjing area the temporal and spatial dynamics of land use change from 1998 to 2006 were studied. Studies have shown that CLUE - S model for the development of the city spatial structure also has the strong ability to predict, to guide urban planning, landscape dynamic driving mechanism have important reference value.

Baiwan qi [11], using the CLUE - S model analyzed the dynamic simulation of land use of dadu river upstream, respectively in 1967 and 1987, the land use spatial data of 1987 and 2000 are simulated by the land use of the space condition, and the land use status of 1987 and 2000 figure is verified, the Kappa value reached 0. 86 and 0. 89.

Zhanghua [12] basing on three different water resources quantity under the constraints of the situation, simulated the land use/land cover change from 2001 to 2020 of zhangye by CLUE - S model.

Peng jian [13] simulated and forecasted in karst area the typical fragile ecological environment basin in four different images of target mode of land use/land cover change by use CLUE -s model.

Li Wen [14] application improved CLUE - S model, in zhenjiang as the study area, first of all, on the basis of the theory and method research of zhenjiang natural, social, economic status, and analyzing the present situation and problems of land use, combined with the basic data of investigation and collection, summarized the influence factors of zhenjiang land use change. 


\section{The constitution of CLUE - S model}

CLUE - S model is mainly composed of rules of transfer of land use, land policy and the restricted areas, land demand and space characteristics of four of input conditions and a space allocation module, four of them is the basis of the normal operation of model input conditions.

Space policy and the restricted areas have a certain influence on the change of land use pattern, the impact of these policies in the CLUE - S model are generally limited land use pattern change. These limiting factors can be divided into two kinds, a kind of to regional restriction factors, such as to establish nature reserves, planning of basic farmland protection areas. Another is policy limiting factors, such as a ban deforestation policies can limit forest land to other land use types.

Land demand through calculation or estimate external model, to limit the total amount of land use type change in the process of simulation.

Land use type transferring setting determines the time of simulation and the power, it includes land use type transfer elastic and land use type transfer order two parts. Elasticity of land use is mainly affected by the change of land use type reversible. Using the high degree of land use type is difficult to take advantage of the low degree of land use type change. On the contrary, the low degree of land use land types are easily to high degree of land use in class. It usually numerical value between 0 and 1, when the value, the greater the likelihood of the closer to 1 the move. Land use type by setting a transfer order defined by the transfer matrix between each land use type can make the transition between various land use type, 1 said can change, 0 means can't change.

Spatial characteristics based on the land use type change in the position of the most likely the theory basis. Is to calculate the various land use types on the spatial distribution of probability, namely the spatial distribution of suitability of various land use types, it is mainly affected by the spatial distribution of factors of the driver. In a CLUE - S model, using Logistic regression to calculate the spatial distribution of the land use type and the driving force, the relationship between the Logistic regression by calculating the probability of occurrence of events, use the independent variables as predicted, can explain the relationship between the land use type and the driving force factors. Its advantage is, variables that can make a continuous can be separated. Its expression is as follows:

$\log \left(\frac{P_{i}}{1-P_{i}}\right)=\beta_{0}+\beta_{1} X_{1 i}+\beta_{2} X_{2 i}+\ldots \ldots+\beta_{\mathrm{n}} X_{\mathrm{n} i}$

Pi says each grid unit may occur the probability of a certain land use type;

$\mathrm{X}$ said the driver;

$\beta$ is the regression coefficient of each influence factor;

Stepwise regression method is helpful to select from many influence factors of the land use pattern correlation is significant factor, the department is not significant to the interpretation of land use pattern will variables are eliminated in the final regression results [12]. Farm the land for each kind of fitting degree of regression equation can be used to test in the ROC curve, according to the size of the area under the curve to determine the class probability to calculate the distribution pattern and is whether has the high consistency between class distribution, the value is between 0.5 to 1 , the more value the class probability distribution of the earth and true to the good consistency between class distribution, the regression equation can better explain to the class of the spatial distribution, the more precise the land use allocation model runtime; Conversely, if the value is close to about 0.5 , shows that the regression equation to explain the significance, the lower class distribution. It is generally believed as the ROC is greater than 0.7 , the identified driver has a good ability to explain. 


\section{CLUE-S Model forecast}

Combining with the above research application, considering the land utilization involves many fields of science such as natural, social and economic problems, the following problems still need further study on the model:

First of all, the space allocation module is the core part of the CLUE - S model, and the output of the model results are also expressed in the form of visual space. However, a recent recipients changes in regional land often mainly influenced by artificial factors, these human driving factors in general could not have space or difficult to space. Therefore, when using CLUE - S model, how to consider many human driving factors, maybe the model should be the perfect place.

Second, the exact relationship between different spatial scale and effect feedback and hidden in the relationship between cause and effect in the process, most are still unknown, how to link to a multiple case study represents the spatial heterogeneity of network, thus the local scale and regional scale of land use change dynamic link, also will be the important research topics in the future.

\section{Acknowledgements}

This work was financially supported by The Technology Research of Huihua College of Hebei Normal University(20140409).

\section{References:}

[1] Huajun Tang, Wenbin Wu, Peng Yang, etc..The Geographical Journal, 2009,64(4):456-468(Tn Chinese).

[2] VerburgPH, Soepboer.W, Encironmental Management,2002,30(3):391-405.

[3] Veldkamp A, Fresco L O. Ecological modeling, 1996, 85(2): 253-270.

[4] Overmars, K.P., Verburg, P.H., Veldkamp, Land Use Policy . 2007. 24(3): 584-599

[5] Verburg, P.H., Eickhout, B., van Meijl, H.. Annals of Regional Science., 2007

[6] Yongzhong Tan, PhD thesis, zhejiang university, 2004(Tn Chinese).

[7] Dissertation Vincent Orekan, Implementation of the local land-use and land-cover change model CLUE-S for Central Benin by using socio-economic and remote sensing data

[8] Castella, J.C., Verburg, P.H. Ecological modeling ,2007. 202: 410-420.

[9] Zengqiang Duan, Fengrong Zhang etc. The Geographical Journal, 2004(Tn Chinese).

[10] Sheng Sheng, Hong Chen etc. Journal of Ecology, 2008(Tn Chinese).

[11] Wan qi Bai, Jian zhong Yan etc. Geography Research, 2005(Tn Chinese).

[12] Hua Zhang, Bo Zhang, etc. Remote Sensing Technology and Application, 2004(Tn Chinese).

[13] Jian Peng, PhD thesis. Beijing university,2006(Tn Chinese).

[14] Wen Li, Nanjing normal university, master's degree thesis, 2011(Tn Chinese). 